\title{
LA DEFORMACIÓN PERMANENTE EN LAS MEZCLAS ASFÁLTICAS Y EL CONSECUENTE DETERIORO DE LOS PAVIMENTOS ASFÁLTICOS EN EL PERÚ
}

\author{
THE PERMANENT DEFORMATION IN THE ASPHALT MIXTURES AND THE \\ CONSEQUENT DETERIORATION OF ASPHALT PAVEMENTS IN PERU
}

\author{
Néstor Huamán Guerrero', Carlos M. Chang Albitres²
}

\section{RESUMEN}

En los últimos años, el Perú ha impulsado una política favorable para la construcción de obras viales a lo largo y ancho del territorio, por lo cual se ejecutó más de 15,000 kilómetros de carreteras con pavimentos asfálticos, según se dice en los reportes del Ministerio de Transportes y Comunicaciones, organismo encargado de la red vial nacional.

Ante esta realidad, existe la imperiosa necesidad de mejorar la tecnología de los pavimentos asfálticos en el Perú, para que alcancen la vida útil para la que fueron diseñados. La deformación permanente es una de las fallas más preocupantes en el deterioro de pavimentos y se hace necesario conocer sus causas fundamentales a fin de tomar las previsiones del caso en las etapas de elaboración del proyecto, construcción y mantenimiento futuros.

Por esto, es primordial que se realicen, en el Perú, diversos ensayos y análisis que utilicen equipos de laboratorio y de campo especializados, los cuales permitan evaluar la estructura del pavimento para evitar la deformación permanente. Esto conduce a la necesidad de desarrollar nuevas especificaciones técnicas para mezclas asfálticas que, dependiendo de los resultados del análisis, puedan incluir el uso de modificadores como polímeros, polvo de caucho y la aplicación de la tecnología SUPERPAVE para una mejor caracterización de los materiales constituyentes de la mezcla asfáltica, con el propósito de incrementar la durabilidad de los pavimentos asfálticos.

Palabras clave: deformación permanente, mezcla asfáltica, comportamiento reológico, ahuellamiento, agrietamiento por fatiga.

\begin{abstract}
In the last years, Peru has promoted a favorable policy for the construction of road works across the entire territory, having executed more than 15,000 kilometers of roads with asphalt pavements, according to reports made by the Ministry of Transport and Communications, the entity responsible for national road network.

Faced with this reality there is an urgent need to improve the technology of asphalt pavements in Peru so that they reach the life for which they were designed. Permanent deformation is one of the failures of most concern in pavement deterioration, being necessary to know their fundmental causes in order to take measures during the development of project, construction, and future maintenance.

Therefore, it is urgent to conduct several tests analysis in Peru using specialized laboratory and field equipments to evaluate the pavement structure and to prevent permanent deformation. This leads to the necessity for developing new technical specifications for asphalt mixes which, depending on the results of the analsys, can include the use of modifiers such as polymers, rubber powder and the application of SUPERPAVE technology for better characterization of the constituent asphalt mixes, with the purpose of increasing the durability of asphalt pavements.
\end{abstract}

Keywords: Permanent deformation, asphalt mixes, rheologic behavior, rutting, cracking due to fatigue.

\footnotetext{
1 Facultad de Ingeniería, Universidad Ricardo Palma. Av. Benavides 5440, Lima, Perú. < nhuamang@hotmail.com>

2 Facultad de Ingeniería, Universidad de Texas en El Paso. 500 W University Ave, El Paso, TX 79968, Estados Unidos. <cchangalbitres2@utep.edu>
} 


\section{INTRODUCCIÓN}

La deformación permanente puede presentarse en las diferentes capas que componen la estructura del pavimento, inclusive a nivel de la subrasante. Las causas que la originan son diversas. Es por ello que la elaboración de un proyecto de pavimentos asfálticos debe ser integral y muy cuidadosa en cuanto a la calidad de los materiales seleccionados, el diseño de la mezcla asfáltica y el diseño estructural del pavimento. Así, se hace necesario realizar un estudio detallado del tráfico, el tipo de suelos que conforman la subrasante, las condiciones de drenaje, el clima y otros factores externos como los procesos constructivos.

En el Perú, como en otros países, la deformación permanente, en sus diferentes formas, es una de las fallas más importantes e incidentes en el comportamiento de los pavimentos asfálticos. Por ese motivo, es importante elaborar un estudio bibliográfico dirigido a identificar las principales causas que generan este tipo de falla, puesto que su conocimiento contribuirá a tomar las precauciones necesarias para evitar el deterioro prematuro de los pavimentos.

\section{ANTECEDENTES GEOGRÁFICOS DEL TERRITORIO PERUANO}

Está demostrado que la durabilidad de los pavimentos asfálticos está influenciada directamente por el clima del lugar donde están ubicados. Además, se deben considerar otros parámetros como la magnitud y frecuencia de las cargas de tránsito, las propiedades de los materiales que lo conforman, las características de la subrasante, la humedad, el proceso constructivo, entre otros. Estos parámetros, en su conjunto, afectan sensiblemente el desempeño del pavimento y su potencial para desarrollar fallas por deformación permanente.

En este contexto, es muy importante considerar que la geografía del Perú es una de las más complejas y diversas. El país se encuentra situado en la parte central y occidental de América del Sur. Está conformado por un territorio de $1.285 .215,60 \mathrm{~km}^{2}$ de superficie continental, lo que representa el $0.87 \%$ del planeta. Este se distribuye en región costeńa, con 136.232,85 km² (10,6\%); región andina, con 404.842,91 km² (31,5\%); y región amazónica, con 754.139,84 km² (57,9\%).

El Perú cuenta con distintos accidentes geográficos que demuestran esa diversidad. El pico más alto, por ejemplo, es el Huascarán, en la Cordillera Blanca, que cuenta con una altura de $6.768 \mathrm{msnm}$. Asimismo, la zona más profunda del país es el cañón de Cotahuasi, que incluso supera al famoso Gran Cañón del Colorado. El río más largo es el río Ucayali, afluente del río Amazonas, con $1.771 \mathrm{~km}$ de longitud. El lago navegable más alto del mundo es el Lago Titicaca, que se ubica entre Perú y Bolivia, con $8.380 \mathrm{~km}^{2}$. Finalmente, la isla más grande del litoral peruano es la Isla San Lorenzo en Callao con $16.48 \mathrm{~km}^{2}$. Se trata del tercer país más grande de Sudamérica.

\section{LA VARIEDAD DE CLIMAS EN EL PERÚ}

El hecho de ubicarse cerca de la línea ecuatorial indicaría que el clima del Perú debería ser eminentemente tropical. Sin embargo, dos factores alteran notablemente su clima. En primer lugar, es importante la existencia de la elevada Cordillera de los Andes, paralela en América del Sur al Océano Pacífico. En segundo lugar, se debe considerar a la fría Corriente Peruana o de Humboldt, que se manifiesta de sur a norte hasta la latitud $5^{\circ}$ y que se interseca con la Corriente del Niño en las costas de Piura y Tumbes hasta la latitud $3.2^{\circ}$, al sur de la línea ecuatorial. Estos accidentes, sumados al anticiclón del Pacífico Sur en esta parte del continente, originan una disminución de las temperaturas pro- 
medio anuales en diez grados centígrados en la costa y una gran variedad de climas simultáneos en todo el país. Ese hecho ha situado al Perú como el país con mayor variedad de climas en el mundo: veintiocho de un total de treinta y dos zonas climáticas posibles.

Definitivamente, esta variedad de climas coexistentes en el Perú hace más compleja la aplicación correcta de la tecnología de los pavimentos asfálticos. Por ello, se constituye en un reto para los estudiosos de esta especialidad. Las ocho regiones naturales del Perú que inciden en las diferentes zonas climáticas son:

* Costa o Chala: desde el Océano Pacífico hasta los $300 \mathrm{~m}$ de altitud desde la frontera de Ecuador hasta la frontera con Chile.

* Yunga: desde los $500 \mathrm{~m}$ de altitud hasta los $2500 \mathrm{~m}$ sobre el nivel del mar.

* Quechua: desde 2500 m hasta $3500 \mathrm{~m}$ de altitud sobre los dos flancos de la cordillera.

* Suni: desde $3500 \mathrm{~m}$ hasta $4100 \mathrm{~m}$ so-

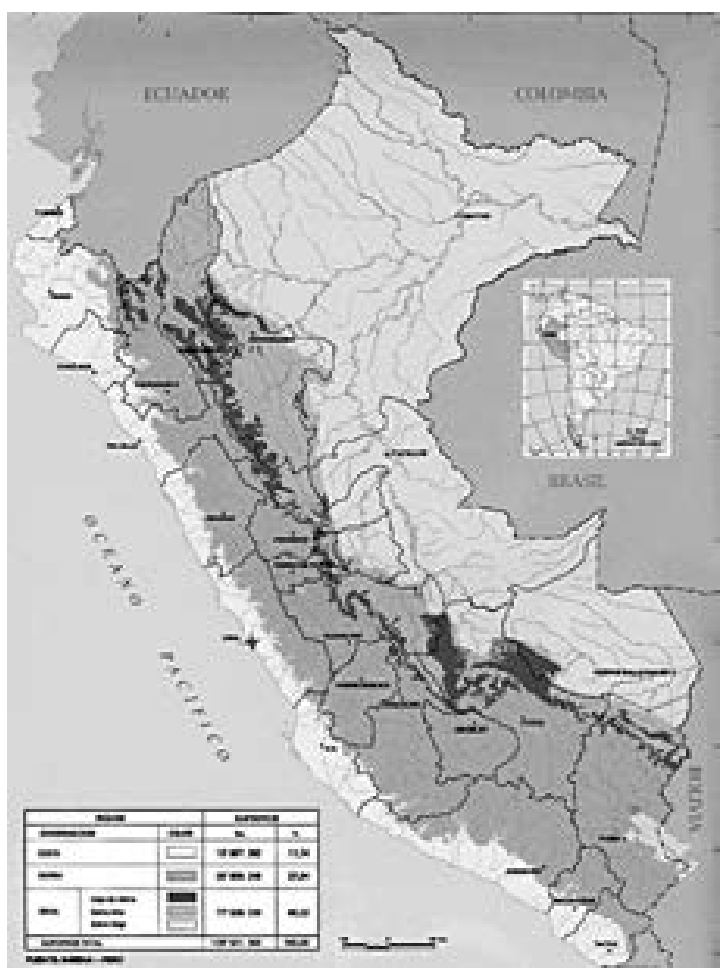

Figura 1. Mapa Geográfico del Perú-Fuente: Wikipedia, la enciclopedia libre bre el nivel del mar.

* Puna: desde 4100 m hasta $4800 \mathrm{~m}$ de altitud. Ocupa el área geográfica de las altas mesetas andinas.

* Jalca o Cordillera: más de 4800m sobre el nivel del mar.

* Selva Alta o Región Rupa Rupa: desde 500 m hasta 1500 m de altitud, pero sobre el flanco oriental de la Cordillera de los Andes.

* Selva Baja o Región Omagua: comprende la gran llanura amazónica, cuyo territorio está por debajo de $500 \mathrm{~m}$.

La figura 1 muestra el mapa del Perú y su ubicación en Sudamérica.

\section{LA DEFORMACIÓN PERMANENTE}

Desde el punto de vista mecanicista, existen dos tipos de falla principales en las mezclas asfálticas, que son la deformación permanente y el fisuramiento por fatiga. La deformación permanente es generada por deformación plástica del concreto asfáltico y/o por deformación de la subrasante.

Cuando a un material granular se le inducen ciclos de carga y descarga, parte de la deformación total $(\xi \mathrm{t})$ que se genera es recuperada y se le conoce como la deformación resiliente $(\xi \mathrm{r})$. Aquella deformación que no se recupera se acumula con cada repetición del ciclo y se le denomina deformación permanente $(\xi \mathrm{p})$. La figura 2 ilustra estos conceptos. 
En un pavimento, estas deformaciones, si son excesivas, generan hundimientos o desplazamientos que se manifiestan en la superficie como ahuellamiento e, incluso, asentamientos de gran magnitud. La deformación "permanente" también se genera por la acumulación de pequeñas deformaciones que ocurren en cada capa, debido a la aplicación de carga, lo cual convierte esta deformación en irrecuperable.

El asfalto es un material que

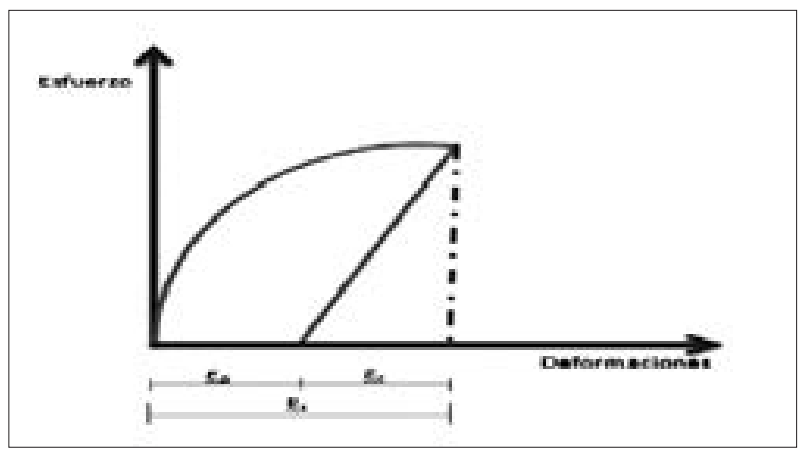

Figura 2. Gráfico ilustrativo sobre la deformación permanente (N. Huamán. 2008) tiene un comportamiento elásticolineal a temperaturas bajas y frecuencias de carga altas, pero muestra propiedades visco-plásticas a temperaturas mayores, por ejemplo, cuando sobrepasan los $40^{\circ} \mathrm{C}$. Debido a este comportamiento, las cargas repetidas del tránsito generan deformación permanente en la capa asfáltica, especialmente cuando es sometida a altas temperaturas, como es el caso de la costa y selva peruanas, cuyas temperaturas del pavimento son mayores a los $60^{\circ} \mathrm{C}$. En carpetas asfálticas, la deformación permanente ocurre debido al flujo viscoelástico o viscoplástico de la mezcla asfáltica. La capacidad de una mezcla de resistir este tipo de deformación depende de diversos factores, entre los cuales se puede considerar la consistencia del ligante asfáltico y la volumetría de la mezcla (agregados y ligantes). El ancho de carriles y la velocidad del tránsito también pueden inducir la deformación permanente. La distribución lateral de la zona de rodadura está influenciada por la velocidad del tránsito, el ancho del carril y la profundidad de las huellas. Las velocidades bajas de tránsito, que corresponden a frecuencias de carga más bajas, también contribuyen directamente al desarrollo de deformaciones permanentes en las capas bituminosas.

Todas las mezclas asfálticas presentan distintas propiedades reológicas que dependen de las propiedades de cada asfalto, la proporción de los diferentes componentes, la distribución del tamańo de las partículas, la angularidad de los agregados y la densidad. Las propiedades de estas mezclas asfálticas también variarán con el tiempo, debido al envejecimiento del ligante. El comportamiento de las mezclas asfálticas con respecto a las deformaciones permanentes depende del tipo de ligante utilizado, la composición de la mezcla, la forma y tamaño de las partículas, la calidad de los agregados, y los aditivos, si son empleados.

La deformación plástica de la mezcla asfáltica tiende a aumentar en climas cálidos y puede darse por una compactación inadecuada de las capas durante la construcción, por el uso de asfaltos blandos o de agregados redondeados.

La temperatura del asfalto del pavimento en servicio es un factor que afecta significativamente a la deformación permanente. No solo las temperaturas máximas, sino también los gradientes de temperatura pueden tener una influencia sobre la deformación permanente.

\section{AHUELLAMIENTO POR FALLA DE LA SUBRASANTE}

Estas deformaciones son causadas por un excesivo esfuerzo repetido en las capas interiores, sean base o subbase. Aunque los materiales "duros" pueden reducir este tipo de ahuellamientos, es considerado un problema como estructura compuesta más que de los materiales en sí. Esencialmente, la estructura de pavimento no es capaz de soportar las cargas actuantes y se deforma. También, el ahuellamiento 


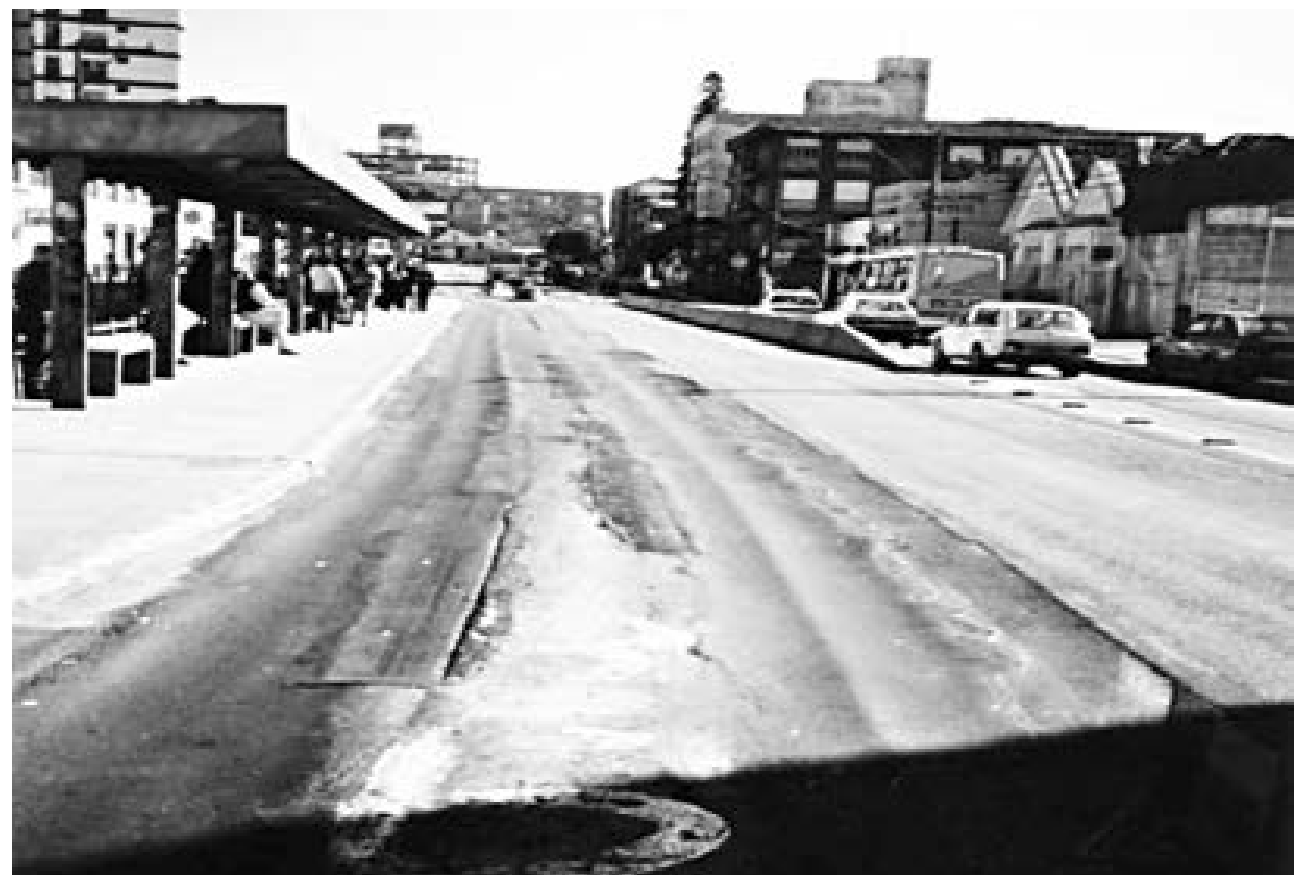

Figura 3. Falla por ahuellamiento de la subrasante. (Bariani L, Goretti L, Pereira J, Barbosa J. 2009)

puede ser causado por el inesperado debilitamiento de una de las capas generadas por la intrusión de humedad que ocasiona la deformación de las capas inferiores, como se observa en la figura 3.

Las cargas excesivas de tráfico provocan debilitamiento de la estructura del pavimento. Los hundimientos por ahuellamiento varían de $750 \mathrm{~mm}$. a $1000 \mathrm{~mm}$. Si se hiciera un corte vertical, la deformación de pavimento indicaría que el espesor del pavimento permanece constante, pero las capas granulares (base o subbase) presentarían deformaciones. Si la estructura del pavimento es muy rígida, en vez de deformarse ocurrirían fisuras por fatiga de un lado a otro en las huellas de los neumáticos.

\section{AHUELLAMIENTO POR FALLA DE LA CAPA DE MEZCLA ASFÁLTICA}

Es el tipo de ahuellamiento que más preocupa a los diseñadores de mezclas asfálticas. Se produce por la incapacidad de una mezcla asfáltica "blanda" de soportar cargas pesadas. Por ello, se acumula la deformación plástica en cada pulso de carga pesada, lo que tiene como resultado el ahuellamiento en la capa de asfalto.

El ahuellamiento, en una mezcla "blanda", ocurre típicamente durante el verano, cuando el pavimento se encuentra sometido a temperaturas de servicio altas que sobrepasan los $60^{\circ} \mathrm{C}$. Esto podría sugerir que las huellas son causadas por los rayos solares, pero es más correcto pensar que se origina por la falta de resistencia de la mezcla asfáltica, que depende de los agregados pétreos y el ligante asfáltico empleados.

El ahuellamiento es producto de la acumulación de pequeñas deformaciones permanentes. Además, para incrementar la resistencia de la capa de mezcla asfáltica, es necesario que se comporte más como un sólido elástico a altas temperaturas. Esto tiene como objetivo que, cuando reciba carga, el material recupere su posición original. Para prevenir el ahuellamiento, es conveniente utilizar as- 
faltos más "duros" y seleccionar agregados de forma cúbica y superficie rugosa que proporcionen un alto grado de fricción interna. Así, cuando la capa de mezcla asfáltica reciba la carga, los agregados y el asfalto trabajarán como un sólido elástico, lo cual les permitirá volver a su forma original cuando se retire la carga. De esta manera, se evitará de esta forma la deformación permanente. Existen equipos de laboratorio y procedimientos de análisis que pueden emplearse durante la etapa de diseño para evaluar si un pavimento es susceptible a la deformación permanente.

A continuación, se presenta una breve discusión referida a lo anterior, con el objeto de anticiparse y prevenir fallas por deformación permanente en pavimentos en servicio.

\section{MEDIDOR DE AHUELLAMIENTO DE RUEDA CARGADA: MARC - PERÚ}

Existe una serie de equipos utilizados que nos permiten evaluar si un diseño de mezcla asfáltica podría presentar deformación permanente en servicio. Con la finalidad de asegurar un mejor comportamiento de las mezclas asfálticas y predecir la respuesta del pavimento ante la presencia de las cargas, se desarrolla, en el Perú, el equipo Medidor de Ahuellamiento de Rueda Cargada (MARC). Este equipo es el resultado de la Tesis de Maestría Desarrollo e Implementación de un Equipo Medidor de Ahuellamiento en Mezclas Asfálticas (Huaya, 2007). El MARC permite medir la resistencia de las mezclas asfálticas en caliente al ahuellamiento y analizar el comportamiento de mezclas con distintos tipos de asfalto y agregados.

Los resultados obtenidos con este equipo demostraron que el ensayo Marshall no es suficiente para caracterizar el comportamiento de las mezclas asfálticas respecto a su resistencia a la deformación permanente o al ahuellamiento. Asimismo, se pudo comprobar que, de los parámetros significativos en la tendencia de la mezcla al ahuellamiento, el más representativo es el contenido de vacíos. El equipo MARC puede, además, medir con mayor claridad la influencia de otros parámetros como las variaciones en la granulometría y textura de los agregados.

\section{RECOMENDACIONES DE LA GUÍA DE DISEÑO DE PAVIMENTOS MECANÍSTICO EMPÍRICO (MEPDG) CON RESPECTO A LA DEFORMACIÓN PERMANENTE}

La metodología empírico-mecanicista para el diseño de pavimentos (MEPDG) de AASHTO, publicada en el 2008, es el resultado de varios años de investigación y su implementación está en proceso en varios países.

Esta nueva guía combina aspectos empíricos y mecanicistas. Los componentes mecanicistas permiten determinar la respuesta del pavimento ante situaciones críticas de cargas de tránsito y clima, utilizando modelos matemáticos para predecir el desempeño. Los componentes empíricos relacionan la respuesta del pavimento predicha con los modelos matemáticos y los indicadores del desempeńo medidos en campo (deterioros e IRI). De esta manera, se verifica que no sobrepasen los límites tolerables definidos para cada indicador y para determinada confiabilidad.

Es así que el procedimiento MEPDG calcula mecanísticamente la respuesta del pavimento (v.g. tensiones, deformaciones y deflexiones) asociadas a cargas de tránsito, condiciones ambientales, y acumula el "daño" producido en la estructura de pavimento durante el período de diseño. Empíricamente, relaciona utilizando funciones de transferencia y modelos de regresión, el daño a través del tiempo con deterioros típicos, como son fisuras, ahuellamiento, e IRI en pavimentos asfálticos, agrietamiento, escalonamiento, e IRI en pavimentos de concreto con juntas, y punzonamiento, ancho de grietas, e IRI en pavimentos de concreto continuamente reforzados. 
Un ejemplo de la calibración del procedimiento de MEPDG, en el caso de Texas, se describe en el trabajo Implementación de la Guía de Diseño Mecanístico de Pavimentos NCHRP 1-37 en Texas (Chang, Freeman, 2007), del que se destaca lo siguiente:

a. Los modelos de respuesta del comportamiento del pavimento usados por la nueva guía de diseño de pavimentos se basan en las características propias del pavimento y modelos representados a través de funciones de transferencia que evalúan la estructura con respecto a la ocurrencia de ciertos tipos de fallas. Estos modelos se utilizan para predecir estados de esfuerzo-deformación en las diferentes capas que componen el pavimento, debido a la acción de cargas de tráfico y condiciones medio-ambientales. Para calibrar estos modelos, se requiere conocer las propiedades de los materiales, las características estructurales del pavimento, la información del tráfico, la condición ambiental, y los datos verificados en campo sobre el comportamiento-respuesta del pavimento. Estos datos deben ser representativos de las condiciones locales de cada región en donde se construirá la estructura de pavimento. Por lo tanto, la calibración y validación de los modelos de comportamiento es crítica para un diseño exitoso. Los modelos de comportamiento estructural involucran fundamentalmente fallas por fatiga $\mathrm{y}$ por ahuellamiento.

b. Las condiciones medio-ambientales tienen un efecto muy importante en el comportamiento del pavimento. Estas condiciones afectan las propiedades mecánicas de los materiales y, por sí mismos, originan esfuerzos de tensión-deforma-

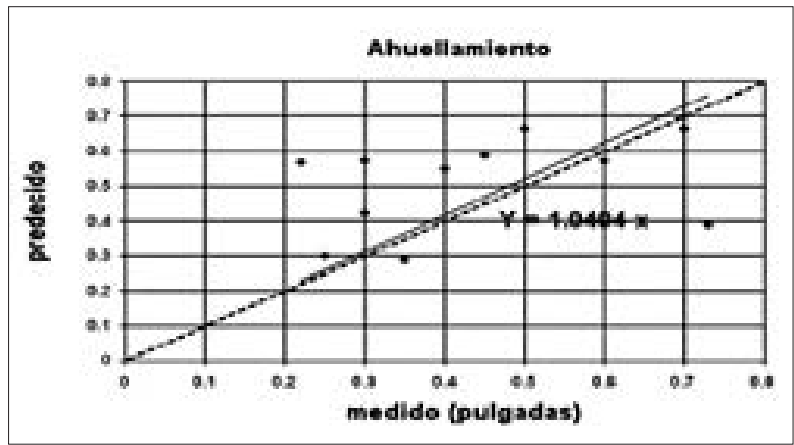

Figura 4. Abuellamiento predecido por los modelos mecanisticos versus mediciones de campo (Chang y Freeman, 2007). ción que conducen a la falla del pavimento. La nueva guía de diseño mecanístico-empírico constituye el primer esfuerzo por considerar, en el análisis del pavimento, los efectos medio-ambientales de una manera mecanística.

c. Un análisis de sensibilidad fue realizado para identificar las variables que tienen mayor influencia en los distintos tipos de falla del pavimento como, por ejemplo, piel de cocodrilo, ahuellamiento, y fisuras longitudinales. La figura 4 muestra una comparación del ahuellamiento predecido por modelos mecanísticos y ahuellamientos medidos en campo.

d. El análisis de sensibilidad realizado indica que las propiedades de las capas del asfalto tienen, comparativamente, una mayor influencia en la respuesta de la estructura del pavimento que las otras capas. Características de la mezcla asfáltica como el módulo de resilencia y la granulometría del agregado son de gran influencia en la respuesta estructural del pavimento.

\section{CONCLUSIONES}

Del trabajo realizado se concluye lo siguiente:

* En el caso de la deformación permanente, se requiere tener especial cuidado en el diseño de la mezcla asfáltica. La selección del tipo de ligante asfáltico debe realizarse de acuerdo a la zona en donde será colocada la mezcla asfáltica para minimizar la influencia de la temperatura y su deformabilidad. Entre menos susceptible a la temperatura sea el ligante asfáltico, más resistente a la 
deformación plástica será la mezcla a altas temperaturas, más aún cuando esta sobrepase los $60^{\circ} \mathrm{C}$. Por ese motivo, se prefieren asfaltos con viscosidad alta y baja susceptibilidad a la temperatura.

* De acuerdo a la geografía del Perú, estas altas temperaturas se presentan en las zonas de selva alta y baja. De la misma manera sucede en la franja costera de aproximadamente 3,000 kms. a lo largo del océano Pacífico, especialmente en la estación del verano, en la que alcanza temperaturas hasta de $40^{\circ} \mathrm{C}$ bajo sombra. Estas elevadas temperaturas hacen que las carpetas asfálticas "trabajen" a temperaturas muy altas, lo que las hace proclives a la falla por deformación permanente de mezcla asfáltica.

* Se considera utilizar los criterios mínimos y máximos acerca del contenido de vacíos en la mezcla, y asegurarlos tanto en la compactación de los especímenes como en la fase de construcción del pavimento. Dentro de los parámetros volumétricos, en ocasiones se recomienda disminuir el contenido de asfalto de las mezclas, mediante el manejo de la granulometría del agregado y valores bajos en el contenido de vacíos. Los contenidos óptimos de asfalto se deben respetar durante la fabricación de la mezcla en planta.

* Como el ahuellamiento en la mezcla asfáltica se produce por la acumulación de pequeńas deformaciones permanentes, se recomienda incrementar la resistencia contra el deslizamiento de las mezclas no sólo utilizando cemento asfáltico más viscoso, sino que el pavimento se comporte más como un sólido elástico a altas temperaturas del pavimento. Así, cuando se aplique la carga, el cemento asfáltico actuará como una banda de goma y volverá a su posición original en lugar de deformarse. En este aspecto, se ha demostrado que el mástico asfáltico que está conformado por el cemento asfáltico y el filler deben recubrir el área de los agregados de manera suficiente para lograr la debida cohesión de la mezcla asfáltica en su conjunto, lo cual también contribuye a evitar el envejecimiento prematuro del asfalto.

\section{RECOMENDACIONES}

Ante la necesidad de evitar que se produzcan fallas por deformación permanente en los pavimentos asfálticos, los autores, con base en las experiencias obtenidas en el desempeńo de la especialidad, recomiendan que se apliquen las siguientes medidas:

1. Tener mayor celo en la selección, diseño, y verificación de la calidad de los agregados con que se fabrican las mezclas asfálticas, considerando que éstos conforman el esqueleto estructural del pavimento y, por lo tanto, su capacidad para resistir las cargas actuantes de los vehículos en tránsito tiene relación directa con la durabilidad del pavimento. Las Especificaciones Técnicas Generales EG 2013 del MTC deben incorporar los resultados de las investigaciones recientes; en particular aquellas referentes a los requerimientos de los agregados por clasificación convencional y Superpave.

2. En cuanto a los cementos asfálticos, si bien es cierto que las EG2013 norman el uso de estos ligantes asfálticos según la temperatura media anual de la zona y en función a su clasificación por penetración, estas normas no son suficientes para aplicarse en proyectos locales. Por este motivo, se recomienda realizar estudios de investigación sobre el uso de asfaltos modificados en departamentos como Loreto, Ucayali, San Martín y Madre de Dios. También, debe considerarse la zona de la selva y toda la zona costera de los departamentos de Tumbes, Piura, Lambayeque, La Libertad, Ancash, Lima, Ica, Arequipa, Moquegua y Tacna. Estas son regiones en las que, por su condición climática, puede hacerse necesario el uso de modificadores (N. Huamán, 2005).

En este contexto, es necesario que en el Perú se clasifiquen también los asfaltos por grado de desempeño (performance grade PG) de acuerdo al Superpave, puesto que la clasificación por 
penetración no evalúa satisfactoriamente el desempeño del asfalto, aún si se considera su comportamiento reológico. Este nos permitirá tener especificaciones técnicas mejoradas que provocarán una mayor durabilidad de los pavimentos asfálticos, puesto que los ligantes asfálticos seleccionados responderán mejor a las exigencias medio-ambientales de la zona donde se ejecutará la obra.

3. Es imprescindible y urgente que las instituciones públicas y privadas del Perú que orientan sus actividades a esta nueva tecnología adquieran equipos de laboratorio que permitan mejorar la selección de los materiales y efectuar diseños de mezcla que aseguren un mejor comportamiento del pavimento ante las deformaciones permanentes. Esto implica la impostergable necesidad de que se actualice la normatividad vigente en la que se incluyan nuevas especificaciones técnicas y nuevos ensayos de laboratorio que permitirán evaluar la utilización de asfaltos modificados con polímeros o con polvo caucho que use el sistema Superpave. Este es un esfuerzo común que requiere de investigación para la adecuación de normas y nuevos ensayos a nuestra propia realidad.

4. Es indispensable que las universidades del Perú que cuentan con facultades de ingeniería civil a nivel de pregrado y posgrado incluyan, en sus programas, cursos de ingeniería de pavimentos, así como que adquieran equipos de laboratorio de última generación que les permitan formar profesionales en esta especialidad a través de trabajos de investigación.

5. Se recomienda la capacitación a nivel nacional e internacional de los profesionales de la actividad pública y privada que trabajan en la especialidad de pavimentos. Esto puede lograrse con el apoyo del Estado, invirtiendo para implementar nuevas tecnologías en nuestro país. Igual actitud debe tomar la empresa privada, puesto que, de esta manera, los pavimentos construidos tendrán un mejor desempeńo, lo que ahorrará inversiones futuras en rehabilitación temprana, y contribuirá al desarrollo económico y social del país.

\section{REFERENCIAS}

Bariani, L., Goretti, L., Pereira, J., y Barbosa, J. (2009). Pavimentación Asfáltica. Formação Básica para Engenheiros. Brasil: s/e.

Chang, C. y Freeman, T. (2007). Implementación de la Guía de Diseño Mecanistico de Pavimentos NCHRP 1-37a en Texas.

Huamán, N. (s/f) La Deformación Permanente en las Mezclas Asfálticas y el consecuente deterioro de los Pavimentos Asfálticos en el Perú. (Tesis para optar el grado académico de Maestro en Ciencias con mención en Ingeniería de Transportes).

Huamán, N. (2005) Los Pavimentos Asfálticos y la Tecnología de Punta. Comunicación presentada en XIII Congreso CILA. Costa Rica.

Huamán, N. (2008). El Estado del Arte de los Pavimentos Asfálticos. Lima: s/e.

Huauya, O., Chang C. (2007). Evaluación del Riesgo Potencial de Ahuellamiento en Mezclas Asfálticas Utilizando el Equipo MARC", Lima: FIC - UNI. 